\title{
Wird die Konjunkturkomponente der Schulden- bremse ihrer Aufgabe noch gerecht?
}

\begin{abstract}
In der Debatte um die Schuldenbremse geht es zumeist um eine Grundgesetzänderung. Dabei ist ein wesentlicher Teil der Schuldenbremse - die Konjunkturkomponente, welche eine Defizitbeschränkung mit antizyklischer Fiskalpolitik vereinen soll - einfachgesetzlich definiert. Sieht man sich diese genauer an, besteht gerade hier Reformbedarf: Die Konjunkturkomponente in ihrer heutigen Ausgestaltung berücksichtigt nicht den neuesten Stand der Wissenschaft, schafft demokratisch fragwürdigen Ermessensspielraum und trägt nur bedingt zu tragfähigen öffentlichen Finanzen bei. Eine einfachgesetzliche Reform der Konjunkturkomponente könnte daher einen pragmatischen Schritt in Richtung einer zukunftsfähigeren Finanzpolitik darstellen.
\end{abstract}

In der zuletzt erschienenen Ausgabe des Wirtschaftsdienst haben wir argumentiert, dass Finanzpolitik heute zukunftsfähig ist, wenn sie die Vollauslastung der Wirtschaft zum Ziel nimmt (Krahé und Sigl-Glöckner, 2021). Denn das Vermeiden von Schulden wirkt zwar zunächst nachhaltig und damit zukunftsfähig. Doch auf den zweiten Blick und im Kontext der heute vor uns liegenden Aufgaben und Umstände, stellt es sich als rückwärtsgewandt und kontraproduktiv heraus. Praktisch folgt daraus, dass eine zukunftsfähige Finanzpolitik heute eine Anpassung der Schuldenbremse erfordert, da diese einer Ausrichtung auf Vollauslastung im Wege steht. Eine Reformmöglichkeit mit niedrigen Hürden wäre eine einfachgesetzliche Änderung der Konjunkturkomponente der Schuldenbremse. Bei genauerer Betrachtung der Konjunkturkomponente zeigt sich, dass ihre Reform nicht nur möglich, sondern nötig ist.

Aufbau der Schuldenbremse und

Konjunkturkomponente

Fiskalpolitische Reformvorschläge werden oft mit der Bemerkung abgetan, dass die Schuldenbremse nun mal im Grundgesetz stehe und es die für eine Verfassungsänderung benötigte Zweidrittelmehrheit in Bundestag und Bundesrat heute nicht gäbe. Tatsächlich sind die Dinge jedoch komplizierter. Art. 115 GG gibt den Rahmen der Schuldenbremse vor. Hier ist festgehalten, dass die zulässige jährliche Nettokreditaufnahme - finanzielle Transaktionen ausgenommen - grundsätzlich auf 0,35\% des

(C) Der/die Autor:in(nen) 2021. Open Access: Dieser Artikel wird unter der Creative Commons Namensnennung 4.0 International Lizenz veröffentlicht (creativecommons.org/licenses/by/4.0/deed.de).

Open Access wird durch die ZBW - Leibniz-Informationszentrum Wirtschaft gefördert.
Bruttoinlandsprodukts (BIP) begrenzt ist. Aber: Eine von der „Normallage“ abweichende konjunkturelle Entwicklung ist bei der Begrenzung der Nettokreditaufnahme (symmetrisch) zu berücksichtigen. Das Verfahren zur Berechnung der maximal zulässigen Nettokreditaufnahme und insbesondere der Berücksichtigung der konjunkturellen Entwicklung (die Konjunkturkomponente, vgl. lila umrahmte Box in Abbildung 1) ist nicht im Grundgesetz festgehalten. Es ist einfachgesetzlich geregelt. Das Ausführungsgesetz zu Art. 115 GG spezifiziert, dass diese auf Basis der Budgetsemielastizität und der Produktionslücke bestimmt werden soll. Die Produktionslücke entspricht der Differenz zwischen dem geschätzten Produktionspotenzial (der Normallage) der Volkswirtschaft und dem erwarteten tatsächlichen BIP.

Darüber hinaus macht das Ausführungsgesetz keine weiteren Angaben zur mathematisch-volkswirtschaftlichen Schätzmethodik des Produktionspotenzials, son-

Dr. Max Krahé ist wissenschaftlicher Mitarbeiter am Institut für Sozioökonomie der Universität Duisburg-Essen und Forschungsdirektor der Denkwerkstatt „Dezernat Zukunft“.

Florian Schuster ist Research Economist bei „Dezernat Zukunft“.

Philippa Sigl-Glöckner ist Direktorin und Geschäftsführerin von „Dezernat Zukunft“. 


\section{Abbildung 1}

Die Struktur der Schuldenbremse

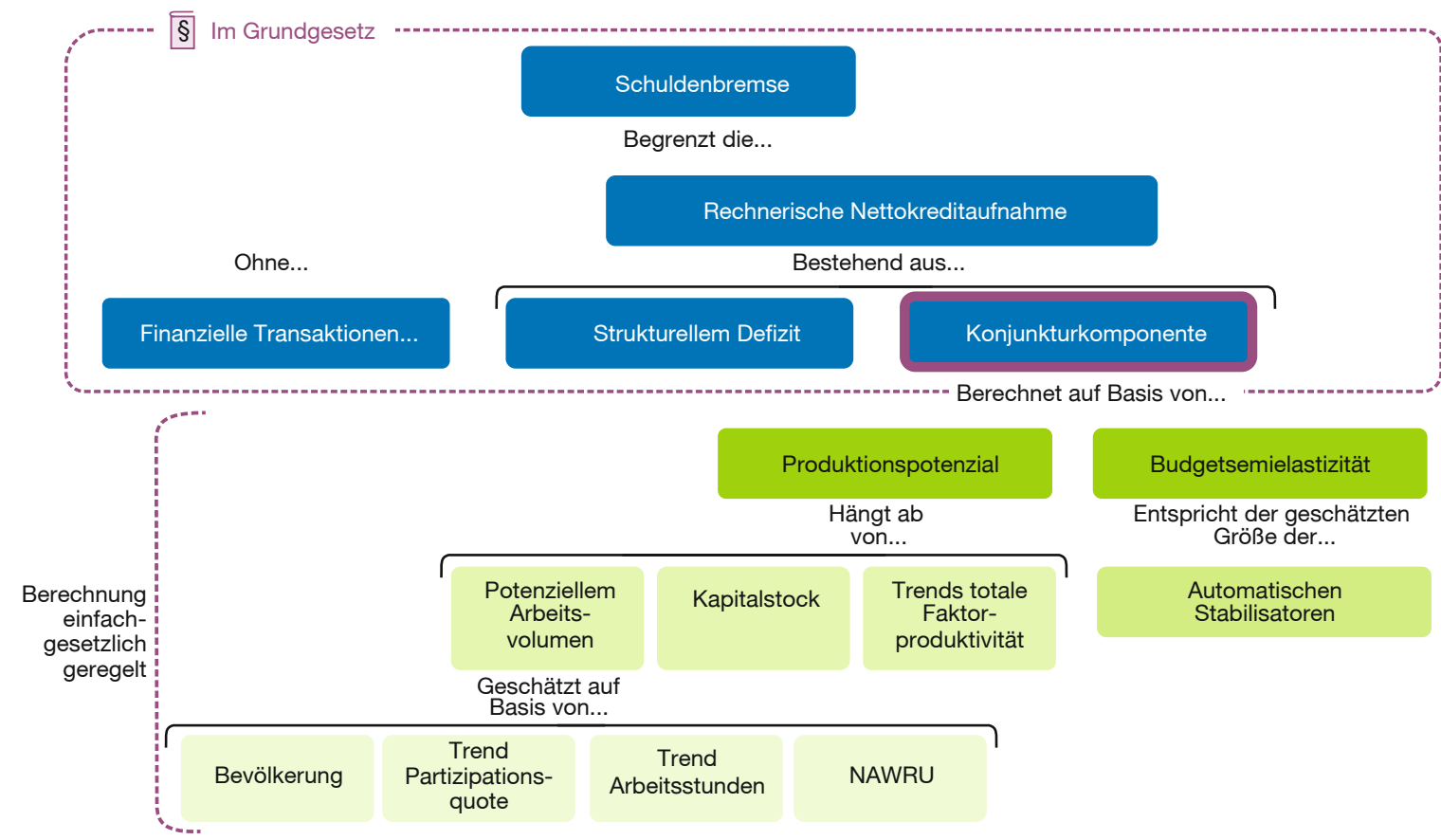

Quellen: BMF, Ademmer et al. (2019), eigene Darstellung.

dern nur zum institutionellen Verfahren, nach dem die Methodik bestimmt werden soll. Laut Abs. 4 sind dafür das Bundesministerium der Finanzen und das Bundesministerium für Wirtschaft und Energie zuständig. Sie haben den Auftrag ,in Übereinstimmung mit dem im Rahmen des Europäischen Stabilitäts- und Wachstumspakts angewandten Konjunkturbereinigungsverfahren“" die Methodik festzulegen und dem letzten Stand der Wissenschaft entsprechend weiterzuentwickeln. Die Verordnung zu Art. 115 legt die Methodik fest. Geschätzt wird das Produktionspotenzial „,mit Hilfe einer gesamtwirtschaftlichen Produktionsfunktion vom Typ CobbDouglas. Über die Produktionsfunktion ergibt sich das Produktionspotential als Kombination aus den normal ausgelasteten Produktionsfaktoren Arbeit und Kapitalstock, multipliziert mit dem Trend der totalen Faktorproduktivität als Maß für den technischen Fortschritt bei Normalauslastung“ (Satz 2 und 3).

Eines spart jedoch auch die Verordnung aus: Die Präzisierung des Begriffs Normalauslastung. Die rechtliche Ausgestaltung der Schuldenbremse, insbesondere der Konjunkturkomponente, stützt sich also erheblich auf Konzepte und Fachbegriffe der ökonomischen Theorie, die sogar Eingang in Gesetzestexte findet. Trotzdem bleibt die Bestimmung der Konjunkturkomponente und damit die Höhe der maximal zulässigen Nettokreditauf- nahme am Ende stark von der Auslegung des Begriffs Normalauslastung abhängig, der im rechtlichen Kontext nicht weiter spezifiziert wird.

Vier Probleme der heutigen Ausgestaltung der Konjunkturkomponente

\section{Mangelnder Einbezug des letzten Forschungsstands}

Das genutzte Verfahren zur Konjunkturbereinigung soll auf Basis des gegenwärtigen Forschungsstands geprüft und weiterentwickelt werden. Doch bereits 2014, vor mittlerweile sieben Jahren, publizierte die Deutsche Bundesbank (2014) ein Papier, das die Zuverlässigkeit von Produktionslückenschätzungen infrage stellte. Problematisch ist unter anderem die Verwendung einer Cobb-DouglasProduktionsfunktion. Diese ignoriert die Nachfrageseite, sie nimmt die Vollauslastung der Kapazitäten schlicht an. Die jüngere Forschung hat jedoch gezeigt, dass eine starke Nachfrage, beispielsweise aus staatlichen Stimuli oder Mittelzuflüssen aus dem Ausland, ebenfalls Potenziale für die gesamtwirtschaftliche Produktion schafft (Mason und Bossie, 2020). Zudem zeigen Gechert et al. (2021) in einer Metastudie von 3.186 Schätzungen aus 121 Studien, dass die Substitutionselastizität von Kapital und Arbeit mit $95 \%$ Wahrscheinlichkeit nicht höher als 0,6 liegt. In der Cobb-Douglas-Produktionsfunktion wird diese mit eins 
angenommen. Die Verwendung der Cobb-Douglas-Produktionsfunktion zur Schätzung des Produktionspotenzials (anstatt des BIP) erfordert außerdem die Definition des Arbeitspotenzials und des Trends der totalen Faktorproduktivität. Die Schätzung des Arbeitspotenzials baut unter anderem auf der NAWRU auf, der niedrigstmöglichen Arbeitslosenquote, bei der es nicht zu sich beschleunigender Lohninflation kommt (vgl. Abbildung 1).

Empirische Evidenz der vergangenen Jahre spricht jedoch dagegen, dass bei Unterschreitung der NAWRU die Inflation ansteigt (z. B. Hazell et al., 2020). Nach Meinung des Federal Reserve Boards (FED) war diese Evidenz sogar so stark, dass das Board sich im Sommer 2020 entschied, sich nicht mehr auf die NAIRU (das US-Äquivalent zur NAWRU) als zentralen Indikator zu stützen. Stattdessen entschied man sich für ein Ziel des maximum employment. Denn „angesichts der dynamischen Natur der Wirtschaft ist es nicht möglich, genau zu wissen, wie weit die Arbeitslosenquote nachhaltig fallen kann, ohne übermäßige Inflation zu verursachen" (eigene Übersetzung, U.S. Federal Reserve, 2021), so die FED auf ihrer eigenen Website. Heimberger et al. bestätigen diese Skepsis: Sie stellen fest, dass die NAWRU-Schätzungen von 1999 bis 2014 erheblich von konjunkturellen Faktoren getrieben wurden, vor allem durch Kapitalakkumulation und Immobilienpreise (Heimberger, Kapeller und Schütz, 2017). Das ist letztlich wenig verwunderlich: Das Gleichungssystem mit dem die NAWRU berechnet wird, isoliert nicht strukturelle Faktoren und ermittelt so die NAWRU, sondern schreibt den historischen Trend fort. Die berechnete, von der Konjunktur bereinigte Arbeitslosenquote hat also weniger mit der Identifikation eines zugrundeliegenden Potenzials als mit dem IstZustand in der jüngeren Vergangenheit zu tun. Gleiches gilt für den Trend der Partizipationsquote und Arbeitsstunden.

Der Trend der totalen Faktorproduktivität, neben Arbeitspotenzial und Kapital der dritte Faktor in der Berechnung des Produktionspotenzials, ist ebenso problematisch. Die Totale Faktorproduktivität (TFP) verkörpert den Teil des BIP-Wachstums, den Arbeitspotenzial (L) und Kapital $(K)$ nicht erklären können $\left(B I P=L^{\alpha} \times K^{1-\alpha} \times T F P\right)$. Sie ist eine Residualvariable. Moses Abramovitz (1956) nennt sie daher a "measure of our ignorance“. Der Trend der totalen Faktorproduktivität ist schlicht die Fortschreibung dessen, was wir nicht wissen.

Darüber hinaus tendieren sowohl die TFP als auch deren Trend zu prozyklischem Verhalten. Laut Field (2007) hat für die US-Wirtschaft zwischen 1890 und 2004 eine Reduktion der Arbeitslosenquote um einen Prozentpunkt zu einem Anstieg des Wachstums der TFP um ca. 0,9 Prozentpunkte geführt. Auch der Trend der TFP hat also wenig mit dem Potenzial der Wirtschaft zu tun und mehr mit einer theore- tisch kaum begründbaren Fortschreibung der Vergangenheit. Um die Produktionspotenzialschätzung wieder in Einklang mit dem aktuellen Stand der Wissenschaft zu bringen, täte eine Weiterentwicklung ihrer Methodik also Not.

\section{Fragwürdige demokratische Legitimation}

Art. $115 \mathrm{GG}$ legt fest, dass eine quantitative Begrenzung der maximal zulässigen Neuverschuldung zu bestimmen ist. Einer der entscheidenden Faktoren im Rahmen der Schuldenbremse, die Definition der Normallage, bleibt jedoch letztlich unbestimmt, da die Normalauslastung der Produktionsfaktoren von der Gesetzgebung nicht definiert wird. Damit wird jenen technokratischen Akteuren, die die Normalauslastung tatsächlich quantifizieren, signifikanter Ermessensspielraum bei der Bestimmung der Neuverschuldungsgrenze eingeräumt. Dieser Ermessensspielraum hat in seiner Größe eine nur fragwürdige Legitimation.

Eine Möglichkeit, diesen Ermessensspielraum einzugrenzen, wäre, die Normalauslastung schlicht als historisches Mittel zu interpretieren. Mit Blick auf die Begriffsgeschichte ist eine solche Interpretation allerdings fraglich. Schon in den 1980er und 1990er Jahren beschränkte sich das damals zur Anwendung kommende Verfahren des Sachverständigenrats (1985) nicht auf die Fortschreibung der Vergangenheit.

Aber selbst wenn man diese Auslegung des Begriffs für gerechtfertigt hält, hilft sie kaum, den Ermessensspielraum technokratischer Akteure zu begrenzen. Die Vergangenheit spricht nicht für sich alleine; sie muss zum Reden gebracht werden. Allein minimale technische Modifikationen, wie eine Veränderung der Varianzbegrenzung der stochastischen Fehlerterme bei der Berechnung der NAWRU, können zu signifikant unterschiedlichen Ergebnissen bei der Fortschreibung der Vergangenheit in die Zukunft führen. So hätte laut Fioramanti (2016) die italienische NAWRU im Herbst 2017 einen ganzen Prozentpunkt niedriger gelegen, wenn die Varianzbegrenzungen von 2014 zum Einsatz gekommen wären. Die heutige Methode zur Berechnung der maximal zulässigen Neuverschuldung krankt also an einem beträchtlichen demokratischen Defizit. Dabei bedarf es laut Korioth (2021) genau hier einer Rechtfertigung: „Jede getätigte oder nicht getätigte Neuverschuldung ist eine politische Entscheidung, die begründet und verantwortet werden muss." Ob die jetzige Methodik diese Verantwortung angemessen platziert scheint ungewiss.

\section{Ursprüngliche Intention der Gesetzgebung nicht mehr gewahrt}

Aber nicht nur das Verfahren zur Berechnung der Konjunkturkomponente an sich ist problematisch. Auch die ur- 
sprüngliche Intention der Gesetzgebung bei der Einführung der Schuldenbremse scheint heute nicht mehr gewahrt. Grund dafür ist die Prioritätensetzung bei der jetzigen Ausgestaltung der Konjunkturkomponente. Das oberste Ziel der heutigen Ausgestaltung ist die Vermeidung dauerhafter Defizite. Eine solche Ausgestaltung kann man als notwendig ansehen, wenn man Art. 115 Abs. 2 Satz 1 GG „Einnahmen und Ausgaben sind grundsätzlich ohne Einnahmen aus Krediten auszugleichen" als Verschuldungsverbot interpretiert. Art. 115 Abs. 2 Satz 3 GG besagt jedoch: „Zusätzlich sind bei einer von der Normallage abweichenden konjunkturellen Entwicklung die Auswirkungen auf den Haushalt im Auf- und Abschwung symmetrisch zu berücksichtigen“, wodurch die Möglichkeit konjunkturstabilisierender, kontrazyklischer Defizite gewährleistet werden soll. Technisch wurde diese Doppelanforderung - konjunkturstabilisierende Defizite ohne dauerhafte Neuverschuldung - umgesetzt, indem sich das Produktionspotenzial am historischen Mittel der Wirtschaftsleistung orientiert. Mit dieser Methodik kann es zwar kurzfristige Produktionslücken geben, eine dauerhafte Produktionslücke schließt die Methodik jedoch aus: Das aktuelle BIP kann nicht dauerhaft unter dem eigenen historischen Mittel liegen.

Damit nimmt man jedoch notwendigerweise in Kauf, dass sich das Produktionspotenzial und damit die Konjunkturkomponente prozyklisch verhält. Läuft die Wirtschaft heute gut, wird das Produktionspotenzial von morgen entsprechend hoch eingeschätzt. Läuft die Wirtschaft heute schlecht, wird das Produktionspotenzial von morgen entsprechend negativ eingeschätzt. Infolgedessen erhöht bzw. reduziert sich die Höhe der Konjunkturkomponente. Das konterkariert die Absicht der Gesetzgebung, prozyklische Fiskalpolitik zu verhindern: „Durch die symmetrische Berücksichtigung der konjunkturellen Auswirkungen auf den Haushalt wird bezweckt, ein pro-zyklisches Verhalten zu vermeiden“ (Fraktionen der CDU/CSU und SPD, 2009, 12). Legt man Art. 115 Abs. 2 GG also als Verschuldungsverbot aus, resultiert das in einem Widerspruch. ${ }^{1}$

Eine kohärentere Interpretation ergibt sich, wenn man dem Wortlaut des Art. 115 GG folgt und die im Gesetzentwurf zur Änderung der Schuldenbremse enthaltene Begründung zu Rate zieht: In dem zitierten Text zu Art. 115 Abs. 2 Satz 3 GG findet sich bei genauem Lesen keine Forderung nach einer Konjunkturkomponente, die durchschnittlich bei null liegt, sondern nur nach der „symmetrischen Berücksichtigung" von Auf- und Abschwüngen. Jeder Euro, um den die Wirtschaft über Potenzial liegt, muss also genauso für die Konjunkturkomponente berücksichtigt werden wie jeder Euro, um den die Wirtschaft unter Potenzial

1 Man könnte außerdem hinterfragen, wieso bei einem generellen Verschuldungsverbot ein strukturelles Defizit von $0,35 \%$ des BIP erlaubt ist. liegt. Dies ist gewährleistet, solange die automatischen Stabilisatoren symmetrisch ausgestaltet sind. ${ }^{2}$ Dass die Berechnungsmethode so auszugestalten ist, dass die Produktionslücke (und damit die Konjunkturkomponente) mathematisch im Durchschnitt über den Zeitverlauf auf null gezwungen wird, steht hingegen nicht im Gesetzestext. Es ist auch nur schwer mit einer plausiblen Interpretation der relevanten Konzepte und der Realität vereinbar.

Eine weitere Interpretationshilfe liefert die Begründung der 2009 vorgenommenen Änderungen für Art. 109 GG und Art. 115 GG, in der erklärt wurde, wieso die Gesetzgebung eine Neujustierung des fiskalischen Regelwerks als nötig erachtete. Diese Begründung gibt Hinweise darauf, wie der Widerspruch zwischen einer anscheinend zwingend prozyklischen, die Verschuldung verhindernden Potenzialschätzung und dem Ziel einer antizyklischen Fiskalpolitik aufgelöst werden kann. Die Begründung definiert den mit der Gesetzesänderung zu adressierenden Missstand als einen Anstieg der Schuldenquote. Was genau daran problematisch ist, wird nicht explizit verbalisiert; implizit scheint es um Generationengerechtigkeit, den Erhalt staatlicher Handlungsmöglichkeit, Wachstums- und Beschäftigungsverluste, Konformität mit dem europäischen Stabilitäts- und Wachstumspakt sowie die nachhaltige Eindämmung der Neuverschuldung an sich zu gehen (Fraktionen der CDU/CSU und SPD, 2009, 5 f.). Ziel der Reform seien Regeln, die „insbesondere in Übereinstimmung mit der Philosophie des präventiven Arms des reformierten europäischen Stabilitäts- und Wachstumspakts die langfristige Tragfähigkeit der Haushalte von Bund und Ländern sicherstellen und nach dieser Maßgabe eine konjunkturgerechte und zukunftsorientierte gestaltende Finanzpolitik ermöglichen sollen“.

Auf Basis der Generationengerechtigkeit lässt sich der Ausschluss dauerhafter Defizite jedoch kaum rechtfertigen: Die Effekte von Defiziten auf die Generationengerechtigkeit hängen vom Verhältnis der Renditen auf Staatsanleihen zum Wachstum ab. „Wenn der Zins [auf Staatsanleihen] unter dem volkswirtschaftlichen Wachstum liegt“, so Blanchard (2019), „dann ist der [durch Verschuldung ausgelöste] Transfer [zwischen Generationen] Wohlstand erhöhend" (eigene Übersetzung). Seit Einführung der Schuldenbremse war das, bis auf die Krisenjahre 2009 und 2020 für die Verschuldung des Bundes immer der Fall.

Außerdem verhindert ein Verbot dauerhafter Defizite eine konjunkturgerechte Fiskalpolitik in Zeiten, in denen die

2 Auch das ist aus Sicht der jüngsten Forschung problematisch, da diese heute asymmetrisch ausgestaltete automatische Stabilisatoren mit semiautomatischen Triggern empfiehlt, z. B. Furman und Summers (2020a). 
Geldpolitik den effektiven Lower Bound erreicht hat. An diesem Punkt hat eine weitere Zinsreduktion eine bremsende Wirkung auf die Wirtschaft. Die Europäische Zentralbank schätzt, dass dies momentan ein Viertel der Zeit der Fall sein könnte, doppelt so häufig wie bei der Einführung des Euro berechnet wurde (Schnabel, 2021). Versäumen Fiskalpolitiker:innen in einer solchen Situation, konjunkturgerechte Defizite zu beschließen, riskieren sie negative Effekte auf Wachstum und Beschäftigung - trotz geringer Verschuldungskosten bzw. sogar staatlicher Gewinne bei Neuverschuldung. Daher folgern Furman und Summers (2020b, 13): „Niedrige Zinsen bedeuten, dass Staaten es sich nicht leisten können, keine fiskalische Expansion zu unternehmen."

Insgesamt ist damit - unter heutigen Umständen - mehr als fraglich, ob die momentane Ausgestaltung der Schuldenbremse und insbesondere der Konjunkturkomponente der ursprünglichen Intention der Gesetzgebung entspricht. Angesichts der deutlich veränderten ökonomischen Umstände, inklusive gefallener Zinsen, niedrigem Wachstum und schwächelnder Inflation, mag das nur wenig verwundern.

\section{Keine europäische Verpflichtung zu dieser Ausgestaltung} der Schuldenbremse

Zielsetzung bei der Einführung der Schuldenbremse war die Konformität mit dem reformierten Stabilitäts- und Wachstumspakt. Daher könnte man argumentieren, dass die Ausgestaltung der Konjunkturkomponente als quantitativer, auf der Produktionslücke aufbauender Automatismus zur Begrenzung der Neuverschuldung alternativlos war. Die EU-Kommission schreibt jedoch weder vor, dass das nötig ist, noch hält sie die Methode zur Berechnung der Produktionslücke dafür geeignet. Wie Buti et al. (2019) schreiben: „Während das fiskalische Überwachungssystem der EU im Kern ein regelbasiertes ist, macht die Gegebenheit, dass es auf einer nicht beobachtbaren Variable [dem Produktionspotenzial] beruht - und unter extremen Umständen zu der Verhängung rechtlich bindender Sanktionen für ein spezifisches Land führen könnte - klar, dass die EU niemals die Intention hatte, dass es für automatische Entscheidungsfindung genutzt werde“ (eigene Übersetzung). So wurde z.B. 2016 das Prinzip des „constrained judgement" eingeführt, das der EU-Kommission die Möglichkeit gibt, unter bestimmten Umständen eine von den Berechnungen abweichende Einschätzung der Produktionslücke zu treffen und für die Umsetzung der europäischen Fiskalregeln zu verwenden (Europäische Kommission, 2019). Zudem sind die Vorgaben zur Einhaltung des präventiven Arms des Stabilitäts- und Wachstumspakts (in dessen Kontext die Schätzung des Produktionspotenzials zur Anwendung kommt) in einigen weiteren Punkten groß- zügiger bzw. flexibler als die der Schuldenbremse, wohl auch um der Problematik der Methodik zur Schätzung des Produktionspotenzials Rechnung zu tragen. Deutschland steht also vor dem Problem, dass sich seine verfassungsrechtliche Fiskalregel auf eine ökonomische Methodik stützt, die für einen anderen Kontext entwickelt wurde und für eine Anwendung wie die im Kontext der Schuldenbremse nicht geeignet ist.

Zwei Vorgehensweisen scheinen möglich, um diesen Missstand zu beheben:

- Deutschland modifiziert seine eigene Schätzung des Produktionspotenzials ohne auf Anpassung der Methodik im Kontext des Stabilitäts- und Wachstumspakts zu drängen, da die Schuldenbremse heute ohnehin restriktiver ist als die europäischen Regeln.

- Deutschland setzt sich im Rahmen der Output Gaps Working Group der EU-Kommission bzw. im Rahmen der bevorstehenden Überprüfung des Stabilitäts- und Wachstumspakts für eine Reform der Schätzung des Produktionspotenzials ein und gleicht die eigene Methodik dann an diese an.

\section{Ein Reformvorschlag}

\section{Mindestkriterien}

Da eine fundamentale Reform der Schuldenbremse oder Methodik zur Berechnung des Produktionspotenzials in der kurzen Frist nicht absehbar ist, schlagen wir als temporäre Lösung vor, die Inputs zur Berechnung des Produktionspotenzials nicht länger arbiträren, annahmenreichen statistischen Verfahren zu überlassen, die notwendigerweise mit erheblichem Ermessensspielraum in der Ausführung einhergehen. Stattdessen könnten diese Inputs auf Basis der folgenden drei Mindestkriterien angepasst werden. Diese Anpassungen reichen zwar kaum aus, um die Berechnung zu legitimieren, sie könnten sie jedoch weniger problematisch machen:

1. Wo immer es keine eindeutige wissenschaftliche Evidenz gibt, sollte der politische Prozess entscheiden. Fachexpertise, idealerweise bereitgestellt durch eine unabhängige Institution, könnte diesen Prozess unterstützen, z.B., indem sie die Implikationen verschiedener Entscheidungsoptionen darlegt.

2. Wo das Verfahren weiterhin auf ökonomischer Theorie und Statistik beruht, sollte es dem neuesten Forschungsstand entsprechen, so wie es von $\S 5$ Abs. 5 des Ausführungsgesetzes zu Art. 115 GG verlangt wird. Stellt der neueste Forschungsstand die genutz- 
te Methodik grundsätzlich infrage, kommt (1) zur Anwendung. ${ }^{3}$

3. Die Inputwerte sollten so wenig wie möglich im Widerspruch zu demokratischem Konsens und anderen Rechten stehen: Wo es bereits einen demokratischen Konsens gibt, sollte dieser Berücksichtigung finden, denn „Finanzrecht ist dienendes Recht“ (Korioth, 2012, 95).

Der folgende Reformvorschlag verändert die auf einer Produktionsfunktion aufbauende Berechnungsmethodik bewusst nicht, um die Hürde zu einer Anpassung so weit wie möglich abzusenken.

\section{Drei Modifikationen der Inputs zur Schätzung des Produktionspotenzials}

\section{Modifikation 1: Von NAWRU zu Vollbeschäftigung}

Angesichts der bereits diskutierten Schwierigkeiten bei der Berechnung der NAWRU schlagen wir eine Vereinfachung vor, die das wirtschaftliche Produktionspotenzial - also die Normallage - dort verortet, wo der allergrößte Teil der Arbeitskräfte beschäftigt ist: bei Vollbeschäftigung. Da es keine allgemeingültige quantitative Definition des Begriffs gibt, ${ }^{4}$ schlagen wir als ersten Proxy vor, die Arbeitslosenquote um den Anteil der Langzeitarbeitslosen zu bereinigen. Friktionelle, kurzfristige Arbeitslosigkeit wird es immer geben. Es widerspricht jedoch dem Konzept einer vollausgelasteten Wirtschaft, wenn es Menschen gibt, die eine sozialversicherungspflichtige Beschäftigung suchen, sich bei der Bundesagentur für Arbeit (BA) gemeldet haben, deren Vermittlungsbemühungen zur Verfügung stehen und trotzdem dauerhaft keine Arbeit finden.

\section{Modifikation 2: Gleichberechtigte Partizipationschancen} im Sinne von Art. 3 GG

Die zweite Modifikation setzt ebenfalls an der quantitativen Dimension des Arbeitsmarkts an, indem die Partizipationsrate von Frauen hinterfragt wird. Nach Zahlen von Eurostat und der BA liegt die Erwerbsquote von Männern seit 2005 konstant über $80 \%$, während die der Frauen um

3 Ein Orientierungspunkt für den "neuesten Forschungsstand“ könnte sein, ob andere öffentliche Institutionen in OECD-Staaten auf Basis wissenschaftlicher Erkenntnis gegenwärtig von der Bundesregierung genutzte Methoden infrage stellen oder abgeschafft haben, wie es z.B. mit der NAIRU - dem US-Äquivalent der NAWRU - vor kurzem geschah.

4 In der Praxis - so z. B. in der deutschen Wirtschaftspolitik seit dem Stabilitätsgesetz von 1967 - wird Vollbeschäftigung bei einer Arbeitslosenquote zwischen $2 \%$ und $3 \%$ verortet, doch ist dieser Wert in der Forschung strittig. phasenweise mehr als 10 Prozentpunkte darunterliegt. ${ }^{5}$ Dieselbe Lücke liegt in Schweden bei nur etwa 3,4, in Finnland bei 3,3 und in Litauen gar nur bei 2,3 Prozentpunkten. In den folgenden Berechnungen wird für die Partizipationsspanne zwischen Männern und Frauen im Lichte der Zahlen aus Nordeuropa ein Zielwert von 3 Prozentpunkten angestrebt. ${ }^{6} 2019$ ergibt sich so eine potenzielle allgemeine Erwerbsquote von 76,6\%, verglichen mit tatsächlich 73,9\% nach den Berechnungen der EU-Kommission. Dieser Wert wird für 2020 und 2021 fortgeschrieben, was angesichts des stetigen Anstiegs der Quoten in den vergangenen 20 Jahren einer konservativen Herangehensweise gleichkommt.

Modifikation 3: Unfreiwillige und nicht notwendige Teilzeit beseitigen

In Deutschland arbeiteten 2019 knapp 12 Mio. Menschen in Teilzeit, mit einer durchschnittlichen wöchentlichen Arbeitszeit von 19,5 Stunden. Aus diesen Zahlen allgemeine Aussagen über das Ausmaß der Unterbeschäftigung in Deutschland abzuleiten ist komplex, weil hier zuverlässige, speziell auf Teilzeitbeschäftigte bezogene Daten über längere Zeiträume fehlen. Jedoch werden im Rahmen der europaweiten Arbeitskräfteerhebung seit einigen Jahren die Gründe der Teilzeitbeschäftigung abgefragt. Wir betrachten unfreiwillige und nicht notwendige Teilzeit als vermeidbar. Nach dieser Definition hätten 2019 47,1 \% der Teilzeitbeschäftigen - oder 5,6 Mio. Menschen - mehr arbeiten können. In den ebenfalls im Rahmen der Arbeitskräfteerhebung aufgenommenen Arbeitszeitwünschen gibt eine Mehrheit der unterbeschäftigten Befragten im Durchschnitt seit einigen Jahren konstant ca. zehn zusätzliche Arbeitsstunden pro Woche als Präferenz an (Statistisches Bundesamt, 2020a). Da dieser Wunsch nicht gänzlich den identifizierten Personen zugerechnet werden kann, wird dieses Szenario lediglich als Obergrenze betrachtet. Auch liegen keine Informationen dazu vor, um wie viele Stunden diejenigen, die wegen familiärer Verpflichtungen nicht Vollzeit arbeiten, im Falle eines ausreichenden Betreuungsangebots ihre Arbeitszeit aufstocken würden. Daher wird in einem konservativ geschätzten Szenario die wöchentliche Arbeitszeit der unfreiwillig oder nicht notwendigerweise Unterbeschäftigten um nur fünf Stunden pro Woche aufgestockt.

5 Die Erwerbsquote oder Partizipationsrate misst den Anteil der Erwerbspersonen, d.h. die Gesamtheit der Erwerbstätigen und Erwerbslosen, an der Erwerbsbevölkerung. Letztere bezeichnet in der Definition der Internationalen Arbeitsorganisation die Bevölkerung im Alter zwischen 15 und 74 Jahren.

6 Ein Schließen der Lücke auf $3 \%$ durch Absenken der männlichen Erwerbsquote schließen wir aus, da die aktive Partizipation von mehr als $80 \%$ der Männer im erwerbsfähigen Alter klar demonstriert, dass mindestens dieser Anteil arbeitswillig und -fähig ist und so der Wirtschaft als potenzielle Arbeitskraft zur Verfügung steht. 
Abbildung 2

Konjunkturkomponenten nach der jetzigen Berechnungsmethodik (BMWi) und Modifikationen

in Mrd. Euro

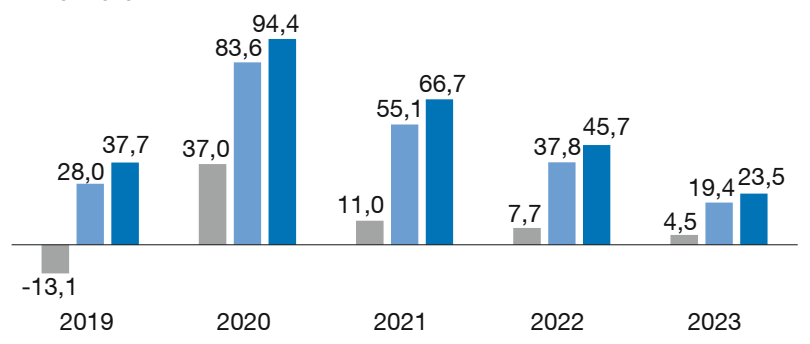

\section{BMWi}

Vollbeschäftigung und Frauenpartizipation und Arbeitsstunden +5

- Vollbeschäftigung und Frauenpartizipation und Arbeitsstunden +10

Quellen: BMWi, eigene Berechnungen.

Die drei beschriebenen Modifikationen ersetzen teils arbiträre, teils ökonomisch zweifelhafte Annahmen und Methoden, die implizit zur Selektion eines Zielpfads für die produktive Kapazität der Wirtschaft beitragen und - so sie andernorts explizit durch die Politik definierten Zielen entgegenlaufen - zu einer widersprüchlichen Politik führen, durch Annahmen, welche die Finanzpolitik in Einklang mit explizit politisch gesetzten Zielen bringen. Damit wird erstens die Finanzpolitik wieder umfassender dem demokratischen Prozess unterworfen, zweitens die Notwendigkeit reduziert, sich auf wissenschaftlich umstrittene Methoden zu verlassen, und drittens die Kohärenz der Politik erhöht. Dabei ist gerade das Letztgenannte ein Faktor, der zur Tragfähigkeit der öffentlichen Finanzen beitragen könnte: Wenn die Politik explizit für die dem Arbeitspotenzial unterliegenden Annahmen verantwortlich ist, steht sie unter dem Druck, entsprechende Maßnahmen vorzulegen, damit dieses Potenzial auch erreicht wird. In Abbildung 2 werden die neuberechneten Konjunkturkomponenten dargestellt. Zusammengefasst schaffen die hier beschriebenen Revisionen der Inputfaktoren zur Schätzung des Produktionspotenzials Spielräume für höhere Defizite, ohne jedoch zu exzessiven oder gar unbegrenzten Verschuldungsspielräumen wie unter der Regelung für Notsituationen zu führen.

\section{Wären die vorgeschlagenen Revisionen im Sinne des Erfinders?}

Ziel der Schuldenbremse ist die Sicherung der langfristigen Tragfähigkeit der öffentlichen Finanzen. Seit 2009 haben sich die Bedingungen dafür wesentlich geändert. Damals mussten noch $12 \%$ des Bundeshaushalts für Zinszahlungen aufgewendet werden (Bundesministerium der Finanzen, 2021). 2020 waren es 1,5\%. Aber während die Kosten der Verschuldung drastisch gefallen sind, be-

\section{Abbildung 3}

Vergleich Zuschuss zur allgemeinen Rentenversicherung und Zinskosten als Anteil des Bundeshaushalts

in $\%$

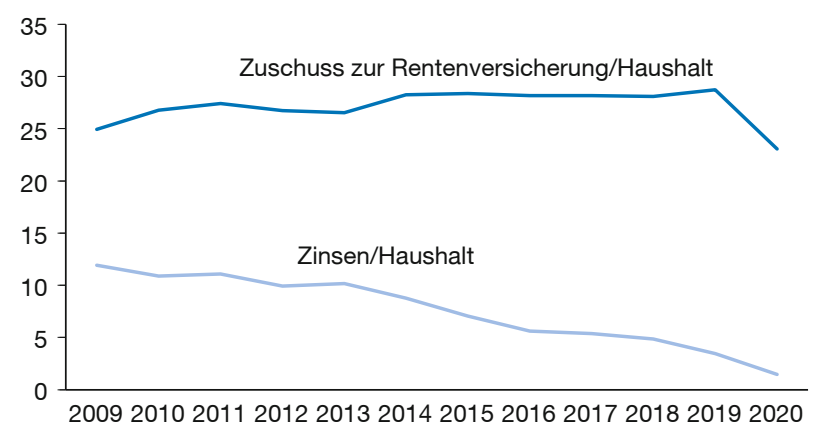

Quelle: BMF.

läuft sich der Zuschuss zur Rentenversicherung auf rund 102 Mrd. Euro oder $23 \%$ des Bundeshaushalts (vgl. Abbildung 3): eine bereits heute beträchtliche Herausforderung. Angesichts der Zunahme des Altersquotienten ${ }^{7}$ von $31 \%$ (2020) auf $49 \%$ (2040) (Statistisches Bundesamt, 2020b) wird diese Herausforderung in den nächsten Jahren an Gewicht zunehmen. Ihre Bewältigung wird wesentlich davon abhängen, dass möglichst viele Menschen im erwerbstätigen Alter eine gut bezahlte und abgesicherte Beschäftigung ausüben, um so ausreichend in die Rentenkasse einzahlen zu können.

Die deutsche Finanzpolitik sollte daher in Zukunft primär auf die Vollauslastung des Arbeitsmarkts und nicht die Vermeidung von Schulden ausgerichtet sein. Dies ist erstrebenswert, nicht weil die Nachhaltigkeit des Bundeshaushalts nachrangig ist, sondern weil ein vollausgelasteter Arbeitsmarkt angesichts des demografischen Wandels und hoher Transferleistungsquoten die Voraussetzung für eine langfristig nachhaltige Finanzpolitik ist.

Fazit

In ihrer heutigen Ausgestaltung erfordert die Schuldenbremse die Quantifikation einer Konjunkturkomponente. Dazu wird die im Grundgesetz erwähnte Normallage als Produktionspotenzial ausgelegt. Die Berechnung des Produktionspotenzials in der heutigen Variante weist jedoch vier Probleme auf: Sie entspricht nicht dem neusten Stand der Wissenschaft; sie schafft einen demokratisch fragwürdigen Ermessensspielraum bei denen, die die Berechnung im Detail umsetzen; sie stimmt in ihren Konsequenzen nicht mehr mit den ursprünglichen Intentionen

7 Verhältnis der Zahl an Personen über 66 zur Zahl an Personen zwischen 22 und 66 . 
der Gesetzgebung überein; und sie ist nicht durch europäische Verpflichtung zu rechtfertigen. Diese ökonomischen, methodischen und politischen Unzulänglichkeiten resultieren letztlich in einer prozyklischen, demokratisch nicht ausreichend legitimierten Fiskalpolitik, ja oft in einer Fiskalpolitik, die im Widerspruch zu anderweitig definierten politischen Zielen steht. Selbst die ursprüngliche Intention hinter der Schuldenbremse, die langfristige Sicherung der Tragfähigkeit der öffentlichen Finanzen, scheint nur bedingt gewahrt.

Der hier präsentierte Vorschlag einer explizit politischen Bestimmung der Inputs zur Berechnung des Produktionspotenzials nach festgelegten Kriterien kann diese Unzulänglichkeiten sicher nicht gänzlich ausräumen. Er könnte aber einen ersten pragmatischen Schritt darstellen, um die problematischsten Aspekte der Schuldenbremse zu reformieren, zur Redemokratisierung der deutschen Fiskalpolitik beizutragen und die Kohärenz zwischen ihr und anderen Politikfeldern wieder zu steigern.

\section{Literatur}

Abramovitz, M. (1956), Resource and Output Trends in the United States since 1870, American Economic Review, 46(2), 5-23.

Ademmer et al. (2019) Schätzung von Produktionspotenzial und -lücke: Eine Analyse des EU-Verfahrens und mögliche Verbesserungen, Kieler Beiträge zur Wirtschaftspolitik, 19, https://www.ifw-kiel.de/de/publikationen/kieler-beitraege-zur-wirtschaftspolitik/schaetzung-vonproduktionspotenzial-und-luecke-eine-analyse-des-eu-verfahrensund-moegliche-verbesserungen-12192/.

Blanchard, O. (2019), Public Debt and Low Interest rates, Working Paper, 19-4, Peterson Institute for International Economics, Februar.

Bundesministerium der Finanzen (2021), Monatsbericht, Mai.

Buti, M. et al. (2019), Potential output and EU fiscal surveillance, VoxEU, 23. September.

Deutsche Bundesbank (2014), On the reliability of international organisations' estimates of the output gap, Monatsbericht, April, 13-35.
Europäische Kommission (2019), Vade Mecum on the Stability \& Growth Pact, Institutional Paper, 101.

Field, A. J. (2007), Procyclical TFP and the Cyclicality of Growth in Output per Hour, 1890-2004, Working Paper, Department of Economics at Santa Clara University, 1.

Fioramanti, M. (2016), Potential Output, Output Gap and Fiscal Stance: Is the EC estimation of the NAWRU too Sensitive to be Reliable?, MPRA Working Paper, 73762

Furman, J. und L. H. Summers (2020a), Automatic Stabilizers in a LowRate Environment, Working Paper, 20-2, Peterson Institute for International Economics, Februar.

Furman, J. und L. H. Summers (2020b), A Reconsideration of Fiscal Policy in the Era of Low Interest Rates, Working Paper, Brookings Institution.

Fraktionen der CDU/CSU und SPD im Bundestag (2009), Entwurf eines Gesetzes zur Änderung des Grundgesetzes (Art. 91c, 91d, 104b, 109, 109a, 115, 143d), Drucksache, 16/12410, 24. März.

Gechert, S. et al. (2021), Measuring capital-labor substitution: The importance of method choices and publication bias, Review of Economic Dynamics.

Hazell, J., J. Herreño, E. Nakamura und J. Steinsson (2020), The Slope of the Phillips Curve: Evidence from U.S. States, NBER Working Paper, 28005.

Heimberger, P., J. Kapeller und B. Schütz (2017), The NAIRU determinants: What's structural about unemployment in Europe?, Journal of Policy Modeling, 39(5), 883-908.

Korioth, S. (2012), § 44 Finanzen, in W. Hoffmann-Riem et al., Grundlagen des Verwaltungsrechts: Band III, C. H. Beck, 2. Aufl.

Korioth, S. (2021), Die Schuldenbremse - reparaturbedürftig?, ifo Schnelldienst, 74(4), 21.

Krahé, M. und P. Sigl-Glöckner (2021), Die Definition einer zukunftsfähigen Finanzpolitik, Wirtschaftsdienst, 101(7), 497-500.

Mason, J. W. und A. Bossie (2020), Public Spending as an Engine of Growth and Equality: Lessons from World War II, Roosevelt Institute.

Sachverständigenrat zur Begutachtung der gesamtwirtschaftlichen Entwicklung (1985), Auf dem Weg zu mehr Beschäftigung, 194.

Schnabel, I. (2021), High Debt, Low Rates and Tail Events: Rules-Based Fiscal Frameworks under Stress, Vortrag, Third Annual Conference des European Fiscal Board, Frankfurt a. M., 26. Februar.

Statistische Bundesamt (Destatis) (2020a), Arbeitszeitwünsche 2019: Knapp 2,1 Millionen Erwerbstätige wollten mehr, 1,5 Millionen wollten weniger arbeiten, Pressemitteilung, 468, 25. November.

Statistische Bundesamt (Destatis) (2020b), Koordinierte Bevölkerungsvorausberechnung für Deutschland -Variante 1: Moderate Entwicklung bei niedrigem Wanderungssaldo (G2L2W1).

U.S. Federal Reserve (2021), What is the lowest level of unemployment that the U.S. economy can sustain?, https://www.federalreserve.gov/ faqs/economy_14424.htm (31. Mai 2021).

Title: Is the Cyclical Component of the Debt Brake Still Up to Its Task?

Abstract: Debates around the German debt brake currently focus on the whether and how of constitutional reform. Yet, a key component of the debt brake - cyclical adjustment - is only specified in ordinary law. And upon closer inspection, it is precisely that part of the debt brake that is most in need of reform: The cyclical component in its current form does not incorporate recent research findings, creates excessive space for discretionary decision making without accountability, and has an ambivalent impact on the sustainability of public finances in the long term. A reform of the cyclical component of the debt brake, which does not require changing the constitution, could therefore be a pragmatic step towards a more sustainable fiscal policy regime in Germany.

JEL Classification: E62, H30, H6O 